\title{
SEMICONTINUITY OF NULLITY OR DEFICIENCY IMPLIES NORMABILITY OF THE SPACE
}

\author{
JOHN M. HOSACK
}

\begin{abstract}
In this paper the upper semicontinuity of nullity and deficiency on locally convex spaces is examined. If either is semicontinuous in the topology of uniform convergence on bounded sets on $L(X)$, then $X$ is normable. If the invertible elements in $L(X)$ are open, then $X$ is normable. The results are applied to topological algebras.
\end{abstract}

Let $X$ be a locally convex Hausdorff linear topological space. Let $L(X)$ be the space of all continuous linear maps from $X$ into $X$ with the topology of uniform convergence on bounded sets. If $T \in L(X)$, define $\tilde{T}: X / \operatorname{ker} T \rightarrow X$ by $\tilde{T}(x+\operatorname{ker} T)=T x$. Let $X / \operatorname{ker} T$ have the quotient topology. There is a $\tilde{T}^{-1}: R(T) \rightarrow X / \operatorname{ker} T . \tilde{T}^{-1}$ is continuous if ond only if (for a normed space) $\gamma(T)=\inf \{|T x| / d(x$, ker $T)\}$, the (reduced) minimum modulus of $T$, satisfies $\gamma(T)>0$, if and only if (for a Banach space) $R(T)$ is closed.

If $X$ is normable, then several results on the semicontinuity of the nullity $\alpha(T)=\operatorname{dim} \operatorname{ker} T$ and the deficiency $\beta(T)=\operatorname{codim} \operatorname{cl}(R(T))$ are known, such as the following result due to Webb.

Proposition [6]. Let $X$ be a normed linear space and $T \in L(X)$ with $\tilde{T}^{-1}$ continuous. Then there is a neighborhood $N$ of $T$ in $L(X)$ such that, for any $A \in N$,

(1) $\alpha(A) \leqq \alpha(T)$,

(2) $\beta(A) \leqq \beta(T)$.

COROLLARY. Let $X$ be a normed linear space. There is a neighborhood $N$ of $I \in L(X)$ such that, for any $A \in N$,

(1) $\alpha(A)=0$,

(2) $\beta(A)=0$.

We shall show that if these conclusions hold then the space $X$ must be normable.

Proposition. Let $X$ be a l.c.s. If there is a neighborhood $N$ of $I \in L(X)$ for the topology of uniform convergence on bounded sets such that for $A \in N$ either $\alpha(A)=0$ or $\beta(A)=0$, then $X$ is normable.

Received by the editors December 13, 1970.

AMS 1969 subject classifications. Primary 4601, 4610, 4650.

Key words and phrases. Normability of locally convex spaces or algebras, semicontinuity of deficiency or nullity, invertible elements. 
Proof. There is a closed, convex, circled and bounded set $B$ and a convex circled neighborhood of zero $V$ such that if $A \in N(B, V)$ $=\{A \in L(X): A B \subseteq V\}$ then either $\alpha(I-A)=0$ or $\beta(I-A)=0$.

Assume if possible that there is an element $x_{0} \in V \backslash B$. Then $x_{0} \neq 0$. By the separation theorems [2] there is a continuous linear functional $m^{\prime}$ on $X$ such that $\left|m^{\prime}(b)\right| \leqq\left|m^{\prime}\left(x_{0}\right)\right|>0$ for all $b \in B$. Set $m(x)$ $=m^{\prime}(x) / m^{\prime}\left(x_{0}\right)$. Then $|m(b)| \leqq 1$ for all $b \in B$ and $m\left(x_{0}\right)=1$. Define $A x=m(x) x_{0} . A \in L(X)$ and since $|m(b)| \leqq 1$ and $V$ is circled, $A b$ $=m(b) x_{0} \in V$ for all $b \in B$. Thus $A \in N(B, V)$. $(I-A) x_{0}=0$, so $\alpha(I-A) \neq 0$. If $(I-A) x=x_{0}$, then $x-m(x) x_{0}=x_{0}, x=(1+m(x)) x_{0}$, and $(I-A) x=0$. Thus $x_{0} \notin R(I-A)$. Since $R(I-A)$ is closed [3], $\beta(I-A) \neq 0$. Thus we must have $V \subseteq B$, so $V$ is a bounded neighborhood of zero, and $X$ is normable.

COROLLARY. $X$ is normable if and only if for every $T \in L(X)$ with $\tilde{T}^{-1}$ continuous there is a neighborhood $N$ of $T$ in $L(X)$ such that for $A \in N$ either

(1) $\alpha(A) \leqq \alpha(T)$ or

(2) $\beta(A) \leqq \beta(T)$.

CoRollary. $X$ is normable if and only if the invertible elements in $L(X)$ are open in the topology of uniform convergence on bounded sets.

It should be noted that perturbations of $I$ may be invertible for operators in some subset of $L(X)$. For example, the following is a corollary of a result of Vladimirskii [5].

Proposition. Let $X$ be a complete l.c.s. Let $U$ be a closed absolutely convex neighborhood of zero. Then there is a closed absolutely convex neighborhood of zero $V$ such that if $\alpha \in L(X)$ is open, $\alpha(U)$ is bounded, and $\alpha(U) \subseteq V$ then $I+\alpha$ is invertible.

Results related to the above corollary have been obtained by Blair [1] and Williamson [7]. $X$ is normable if and only if there is a subalgebra $A$ of $L(X)$ containing all operators of finite rank such that multiplication $A \times A \rightarrow A$ is continuous for the topology of uniform convergence on bounded sets, compact sets, or pointwise convergence on $A . X$ is normable if and only if there is a linear set $L$ in $L(X)$ containing all operators of finite rank which can be given a linear topology such that $(T, x) \rightarrow T x$ is continuous.

The following application to topological algebras (locally convex topological rings with identity) was suggested by this paper's referee. A topological algebra $X$ has a continuous inverse [4] if there is a neighborhood $W$ of the identity $e$ such that every element of $W$ has an 
inverse and the mapping $x \rightarrow x^{-1}$ is continuous on $W$. In this case the invertible elements are open and $x \rightarrow x^{-1}$ is continuous. The left regular representation of $X$ is $R: X \rightarrow L(X)$ defined by $R(a) x=a x$.

Proposition. Let $X$ be a topological algebra whose left regular representation contains the operators of finite rank. If $X$ has a continuous inverse, then $X$ is normable.

Proof. The map $a \rightarrow R(a)$ gives $R(X)$ the topology of pointwise convergence. There is a neighborhood $U$ of $I=R(e)$ for the stronger topology of uniform convergence on bounded sets such that $U \cap R(X)$ $\subseteq R(W)$, whose elements are invertible. Thus there is a closed, convex, circled and bounded set $B \subseteq X$ and a convex circled neighborhood $V$ of 0 such that $I+N(B, V) \cap R(X) \subseteq R(W)$. If there is an $x_{0} \in V \backslash B$, then as before there is a continuous linear functional $m$ such that $|m(B)| \leqq 1$ and $m\left(x_{0}\right)=1$. If $A x=m(x) x_{0}$, then $-A \in N(B, V)$ $\cap R(X)$ and $(I-A) x_{0}=0$. Thus $I-A \in R(W)$ is not invertible, and so $V \subseteq B$ and $X$ is normable.

Applying the left regular representation to the results of Blair and Williamson [7], we obtain the following result.

Proposition. Let $X$ be a topological algebra whose left regular representation contains the operators of finite rank. Then $X$ is normable if and only if multiplication $X \times X \rightarrow X$ is continuous.

\section{REFERENCES}

1. A. Blair, Continuity of multiplication in operator algebras, Proc. Amer. Math. Soc. 6 (1955), 209-210. MR 16, 935.

2. R. E. Edwards, Functional analysis. Theory and applications, Holt, Rinehart and Winston, New York, 1965. MR 36 \#4308.

3. M. A. Gol'dman and S. N. Kračkovskil, Perturbation of homomorphisms by operators of finite rank, Dokl. Akad. Nauk SSSR 174 (1967), 743-746= Soviet Math. Dokl. 8 (1967), 670-673. MR 35 \#7164.

4. M. A. NaImark, Normed rings, GITTL, Moscow, 1956; English transl., Noordhoff, Groningen, 1964. MR 19, 870; MR 34 \#4928.

5. Ju. N. Vladimirskir, $\Phi_{+}$-operators in locally convex spaces, Uspehi Mat. Nauk 23 (1968), no. 3 (141), 175-176. (Russian) MR 37 \#3396.

6. J. H. Webb, Perturbation theory for a linear operator, Proc. Cambridge Philos. Soc. 63 (1967), 11-20. MR 34 \#3340.

7. J. H. Williamson, Two conditions equivalent to normability, J. London Math. Soc. 31 (1956), 111-113. MR 17, 1111.

University of California, Irvine, California 92664 